\title{
HEDGING BY SEQUENTIAL REGRESSION: AN INTRODUCTION TO THE MATHEMATICS OF OPTION TRADING
}

\author{
BY H. FÖLLMER and M. SCHWEIZER
}

ETH Zürich

\section{INTRODUCTION}

It is widely acknowledge that there has been a major breakthrough in the mathematical theory of option trading. This breakthrough, which is usually summarized by the Black-Scholes formula, has generated a lot of excitement and a certain mystique. On the mathematical side, it involves advanced probabilistic techniques from martingale theory and stochastic calculus which are accessible only to a small group of experts with a high degree of mathematical sophistication; hence the mystique. In its practical implications it offers exciting prospects. Its promise is that, by a suitable choice of a trading strategy, the risk involved in handling an option can be eliminated completely.

Since October 1987, the mood has become more sober. But there are also mathematical reasons which suggest that expectations should be lowered. This will be the main point of the present expository account. We argue that, typically, the risk involved in handling an option has an irreducible intrinsic part. This intrinsic risk may be much smaller than the a priori risk, but in general one should not expect it to vanish completely. In this more sober perspective, the mathematical technique behind the Black-Scholes formula does not lose any of its importance. In fact, it should be seen as a sequential regression scheme whose purpose is to reduce the $a$ priori risk to its intrinsic core.

We begin with a short introduction to the Black-Scholes formula in terms of currency options. Then we develop a general regression scheme in discrete time, first in an elementary two-period model, and then in a multiperiod model which involves martingale considerations and sets the stage for extensions to continuous time. Our method is based on the interpretation and extension of the Black-Scholes formula in terms of martingale theory. This was initiated by Kreps and Harrison; see, e.g. the excellent survey of HARRISON and PLISKA $(1981,1983)$. The idea of embedding the Black-Scholes approach into a sequential regression scheme goes back to joint work of the first author with D. Sondermann. In continuous time and under martingale assumptions, this was worked out in SCHWEIZER (1984) and FöLlMER and SONDERMANN (1986). SCHWEIZER (1988) deals with these problems in a general semimartingale model.

The present paper is a written version, with some extensions, of an expository talk given at the annual meeting of the Vereinigung Schweizerischer Versicherunsmathematiker in September 1987. As in the talk, our purpose is to provide an elementary introduction to some key features of the mathematical theory of option pricing, with special emphasis on the use of linear regression. 


\section{FROM HUYGENS AND BERNOULLI TO THE FORMULA OF BLACK AND SCHOLES}

Consider a call option on US dollars against Swiss francs. This option gives the right to buy a specified amount of dollars, say $\$ 100$, at a specified time $T$ at a predetermined exchange rate $K$. If $X_{t}$ denotes the exchange rate, i.e. the value in SFR of $\$ 100$ at time $t \in[0, T]$, then the value $V_{T}$ of the option at the terminal time $T$ will be

$$
H=\left(X_{T}-K\right)^{+}= \begin{cases}X_{T}-K & \text { if } X_{T} \geqslant K \\ 0 & \text { otherwise. }\end{cases}
$$

It is now natural to ask: What is a fair price for this option? In other words: What is the value $V_{0}$ of the option at the initial time 0 when the final outcome $H$ is still uncertain?

Until 1973, when the fundamental papers of BLACK and SCHOLES (1973) and Merton (1973) appeared, there seemed to be an obvious answer. This answer could have been given already by CHR. HUYGENS (1657) and BERNOULLI (1713). To begin with, the exchange rate $X_{T}$ and, consequently, the return $H$ of the option should be viewed as random variables on some probability space $(\Omega, \mathscr{T}, P)$ which describes the possible time evolutions of the exchange rate and their respective probabilities. Given such a stochastic model, the fair price of the option should be equal to the expected value $E[H]$ of the random variable $H$ :

$$
V_{0}=E[H] \text {. }
$$

One could think of several modifications. For example, one could take into account an interest rate $r$ and replace (2.1) by

$$
V_{0}=\frac{1}{1+r} \cdot E[H] \text {. }
$$

Also, the price could include a risk premium, and it would seem reasonable to compute such a premium in terms of the variance $\operatorname{Var}[H]$ of the random variable $H$, since this variance would appear to be a natural measure of the risk involved in handling the option. In essence, however, the problem would have seemed to be reduced to the choice of a suitable probability measure $P$.

Such a probability measure $P$ on the space $\Omega$ of all continuous time evolutions $\omega:[0,1] \rightarrow \mathbf{R}$ was proposed in 1900 by Bachelier in his thesis 'Théorie de la Spéculation'; a rigorous construction was give by Wiener in 1923. Under this Wiener measure $P$, the stochastic process $X_{t}(\omega):=\omega(t)(0 \leqslant t \leqslant T)$ behaves like a Brownian motion. This stochastic model has turned out to be of basic importance, not only for its fundamental connection to physics, but also on purely mathematical grounds. It is, for example, the natural reference model for functional versions of the central limit theorem.

In view of applications to the stock market one might want to use a modified version. For example, SAMUELSON (1964) has proposed to model the stochastic process $X_{t}(0 \leqslant t \leqslant T)$ as the solution of a stochastic differential equation

$$
\mathrm{d} X_{t}=\sigma \cdot X_{t} \mathrm{~d} B_{t}+\mu \cdot X_{t} \mathrm{~d} t
$$


where $\left(B_{t}\right)$ is a Brownian motion. Here $\mu \cdot X_{t} \mathrm{~d} t$ is a trend forecast with drift parameter $\mu$, and $\sigma \cdot X_{t} \mathrm{~d} B_{t}$ is a random fluctuation with volatility parameter $\sigma$. Suppose now that we accept (2.3) as a mathematical model for the stochastic time evolution of our exchange rate $X_{t}(0 \leqslant t \leqslant T)$. In this model, the random variable $X_{T}$ has a log-normal distribution, and the Huygens-Bernoulli prescription (2.1) would lead to formula

$$
\begin{aligned}
V_{0} & =E[H] \\
& =\frac{1}{\sqrt{2 \pi}} \cdot \int_{-\infty}^{\infty}\left(X_{0} \cdot \exp \left(\sigma \cdot \sqrt{T} \cdot u+\left(\mu-\frac{1}{2} \cdot \sigma^{2}\right) \cdot T\right)-K\right)^{+} \cdot \exp \left(-\frac{u^{2}}{2}\right) \mathrm{d} u .
\end{aligned}
$$

But in the same model (2.3), the Black-Scholes formula gives a quite different answer. It tells you that the drift parameter $\mu$ is completely irrelevant, that you might as well replace $P$ by the measure $P^{*}$ corresponding to $\mu^{*}=0$ which makes the exchange rate behave like a fair game, and that the fair price of the option should be computed as the expected value of $H$ in this new model, even though the experts' forecast is given by $P$ and not by $P^{*}$ :

$$
\begin{aligned}
V_{0} & =E^{*}[H] \\
& =\frac{1}{\sqrt{2 \pi}} \cdot \int_{-\infty}^{\infty}\left(X_{0} \cdot \exp \left(\sigma \cdot \sqrt{T} \cdot u-\frac{1}{2} \cdot \sigma^{2} \cdot T\right)-K\right)^{+} \cdot \exp \left(-\frac{u^{2}}{2}\right) \mathrm{d} u \\
& =X_{0} \cdot \Phi\left(\frac{1}{\sigma \cdot \sqrt{T}} \cdot\left(\log \frac{X_{0}}{K}+\frac{1}{2} \cdot \sigma^{2} \cdot T\right)\right)-K \cdot \Phi\left(\frac{1}{\sigma \cdot \sqrt{T}} \cdot\left(\log \frac{X_{0}}{K}-\frac{1}{2} \cdot \sigma^{2} \cdot T\right)\right),
\end{aligned}
$$

where $\Phi$ denotes the cumulative distribution function of a standard normal distribution $N(0,1)$. Also, it is claimed that there is a trading strategy which requires the initial investment $V_{0}=E^{*}[H]$ and then duplicates the contingent claim $H(\omega)$ without any additional cost, no matter which time evolution $\omega \in \Omega$ is realized by the random mechanism described by $P$. In particular, there is no reason to modify the fair price $V_{0}=E^{*}[H]$ by a risk premium.

If we include interest rates in the model, then (2.5) is replaced by

$$
\begin{aligned}
V_{0}= & E^{*}\left[\left(X_{T}-K \cdot \mathrm{e}^{-\rho \cdot T}\right)^{+}\right] \\
= & X_{0} \cdot \Phi\left(\frac{1}{\sigma \cdot \sqrt{T}} \cdot\left(\log \frac{X_{0}}{K}+\left(\rho+\frac{1}{2} \cdot \sigma^{2}\right) \cdot T\right)\right) \\
& -K \cdot e^{-\rho \cdot T} \cdot \Phi\left(\frac{1}{\sigma \cdot \sqrt{T}} \cdot\left(\log \frac{X_{0}}{K}+\left(\rho-\frac{1}{2} \cdot \sigma^{2}\right) \cdot T\right)\right) .
\end{aligned}
$$

Here $\rho$ denotes the continuously compounded rate of return on a Swiss franc account. 
A rigorous mathematical justification of this counter-intuitive prescription involves rather advanced tools, e.g. the Cameron-Martin-Maruyama-Girsanov formula and a deep representation theorem of K. ITô (1951). This explains some of the mystique around the Black-Scholes formula. But from an economic point of view, its crucial feature can already be explained in a very elementary setting which does not require any mathematical sophistication. To this end, we employ the well-known didactic device of using an example with binary structure.

Suppose that the current exchange rate is given by $X_{0}=135$. Consider a call option with a strike of $K=145$ at time $T$. We assume the following binary scenario: the exchange rate at time $T$ will be 175 with probability $p$ or 80 with probability $1-p$. Correspondingly, the return $H$ of the option will be 30 with probability $p$ and 0 with probability $1-p$. Taking into account an interest rate $r$, the Huygens-Bernoulli prescription (2.2) would compute the fair price of the option as

$$
V_{0}=E\left[\frac{H}{1+r}\right]=\frac{1}{1+r} \cdot p \cdot 30
$$

for $p=0.5$ and $r=0.05$ we would get $V_{0}=14.3$. The Black-Scholes prescription, however, would be the following. First replace $p$ by $p^{*}$ so that the exchange rate, properly discounted, behaves like a fair game:

$$
X_{0}=E^{*}\left[\frac{X_{\mathrm{T}}}{1+r}\right]
$$

or, more explicitly,

$$
135=\frac{1}{1+r} \cdot\left(p^{*} \cdot 175+\left(1-p^{*}\right) \cdot 80\right) .
$$

Now compute the fair price as the expected value of the return $H$, properly discounted, in this new model:

$$
V_{0}=E^{*}\left[\frac{H}{1+r}\right]=\frac{1}{1+r} \cdot p^{*} \cdot 30 ;
$$

for $r=0.05$ we would get $p^{*}=0.65$ and $V_{0}=18.6$.

At first sight, this change of the model seems completely arbitrary, just as in the more intricate model above. But in the present simple case we can give a direct economic justification. Suppose that at time 0 you sell the option. Then you can prepare for the resulting contingent claim at time $T$ by using the following strategy:

Sell the option at the Black-Scholes price $18.6+18.6$

Buy $\$ 31.6$ at the present exchange rate of $1.35 \quad-42.7$

Take a loan of SFR 24.1 with interest rate $r=0.05+24.1$ 
Thus, the balance at time 0 is 0 . At time $T$ we have to distinguish two cases:

(i) The dollar has risen: Option is exercized $\quad-30$

Sell dollars at $1.75+55.3$

Pay back loan with interest -25.3

(ii) The dollar has fallen: Option expires 0

Sell dollars at $0.80+25.3$

Pay back loan with interest -25.3

This demonstrates that the Black-Scholes price 18.6 is just the right amount which is needed in order to hedge the option without any risk. Any option price different from the Black-Scholes price would enable either the option seller or the option buyer to make a sure profit without any risk: There would be an arbitrage opportunity.

In the next section, we explain how the correct hedging strategy can be found in a systematic way. In the preceding example it is clear that the model is too simplistic: there is no reason to restrict our attention to a binary scenario. Therefore, we are going to admit more general models. But this will force us to lower our expectations to a more realistic level. In fact, the situation becomes less pleasant as soon as we admit a third possibility for the value of $X_{T}$ : It is no longer possible to reduce the risk to 0 .

From the point of view of the continuous-time model (2.3), a binary situation as above should only serve as an infinitesimal building stone and not be taken seriously in itself. But also on the mathematically much more advanced level (2.3) there are good reasons to believe that the model, and in particular its promise of risk-free option trading, is too nice to be realistic.

\section{HEDGING IN A TWO-PERIOD MODEL: AN EXERCISE IN LINEAR REGRESSION}

Let us first consider a simple two-period model where the exchange rates $X_{k}$ at the initial time $k=0$ and the terminal time $k=1$ are random variables on some probability space $(\Omega, \mathscr{F}, P)$. At time 0 the exchange rate $X_{0}$ is known and can be treated as a constant, i.e. we assume that $P\left[X_{0}=x_{0}\right]=1$ for some $x_{0}>0$. The option, or rather the resulting contingent claim at time 1 , is described by a random variable $H$ defined on the same probability space. For a call option with a strike of $K$, we would have $H=\left(X_{1}-K\right)^{+}$. To keep the exposition as simple as possible, we leave interest rates aside for the moment.

Let us now assume that we have sold the option. At time 1 we will have to pay the random amount $H(\omega)$. We should like to insure ourselves against this event; we want to hedge the option. To this end, we buy $100 \cdot \xi$ dollars and put aside 
$\eta_{0}$ Swiss francs. This initial portfolio at time 0 has the value

$$
V_{0}=\xi \cdot X_{0}+\eta_{0} \text {. }
$$

At the terminal time 1, we want a portfolio whose value is exactly equal to $H$. The value of the dollar account will be $\xi \cdot X_{1}$, and if we then adjust the Swiss franc account from $\eta_{0}$ to $\eta_{1}=H-\xi \cdot X_{1}$, the value

$$
V_{\mathbf{1}}=\xi \cdot X_{1}+\eta_{1}
$$

of the resulting portfolio at time 1 will satisfy our condition

$$
V_{\mathrm{l}}=H \text {. }
$$

For a given $H$, such a strategy will be determined by our initial choice of the constants $\xi$ and $V_{0}$.

Let us examine the costs induced by such a strategy $\left(\xi, V_{0}\right)$. If $C_{k}$ denotes the cumulative cost up to time $k$, then we have

$$
C_{0}=V_{0},
$$

and the additional cost due to our adjustment of the Swiss franc account at time 1 is given by

$$
\begin{aligned}
C_{1}-C_{0} & =\eta_{1}-\eta_{0} \\
& =\left(V_{1}-\xi \cdot X_{1}\right)-\left(V_{0}-\xi \cdot X_{0}\right) \\
& =V_{1}-V_{0}-\xi \cdot \Delta X
\end{aligned}
$$

where we put $\Delta X:=X_{1}-X_{0}$. Let us now choose our trading strategy $\left(\xi, V_{0}\right)$ in such a way that the remaining risk at time 0 , measured by the expected quadratic cost

$$
\begin{aligned}
R & :=E\left[\left(C_{1}-C_{0}\right)^{2}\right] \\
& =E\left[\left(H-V_{0}-\xi \cdot \Delta X\right)^{2}\right],
\end{aligned}
$$

is minimized. This is, of course, a well-known problem: we are simply looking for the best linear estimate of $H$ based on $\Delta X$. Thus, the optimal constants $\xi$ and $V_{0}$ are given by

$$
\xi=\frac{\operatorname{Cov}(H, \Delta X)}{\operatorname{Var}[\Delta X]}=\frac{\operatorname{Cov}\left(H, X_{1}\right)}{\operatorname{Var}\left[X_{1}\right]}
$$

and

$$
V_{0}=E[H]-\xi \cdot E[\Delta X] .
$$

This optimal value $V_{0}$ of the initial portfolio may be regarded as a fair price of the option. In particular we obtain the condition

$$
C_{0}=E\left[C_{1}\right] \text {. }
$$

This means that the optimal strategy is mean-self-financing: once we have determined the initial value $V_{0}=C_{0}$, the additional cost $C_{1}-C_{0}$ is a random variable with expectation $E\left[C_{1}-C_{0}\right]=0$. By this optimal trading strategy, the remaining 
risk is reduced to the minimal mean square prediction error

$$
\begin{aligned}
R_{\min } & =\operatorname{Var}[H]-\xi^{2} \cdot \operatorname{Var}\left[X_{1}\right] \\
& =\operatorname{Var}[H] \cdot\left(1-\left(\rho\left(H, X_{1}\right)\right)^{2}\right)
\end{aligned}
$$

where $\rho$ denotes the correlation coefficient. This value $R_{\min }$ may be viewed as the intrinsic risk of the option $H$. It is this intrinsic risk, and not the a priori risk measured by the variance $\operatorname{Var}[H]$ of $H$, on which any adjustment of the fair price $V_{0}$ by a suitable risk premium should be based.

In a model with an interest rate $r$ on the Swiss franc account, we would have to replace $(3.5)$ by

$$
\begin{aligned}
C_{1}-C_{0} & =\eta_{1}-(1+r) \cdot \eta_{0} \\
& =(1+r) \cdot\left(\frac{V_{1}}{1+r}-V_{0}-\xi \cdot\left(\frac{X_{1}}{1+r}-X_{0}\right)\right) .
\end{aligned}
$$

The optimal choice of $\xi$ would still be (3.7); $V_{0}$, however, is then given by

$$
V_{0}=E\left[\frac{H}{1+r}\right]-\xi \cdot E\left[\frac{X_{1}}{1+r}-X_{0}\right] .
$$

Typically, the crucial quantities $\xi, V_{0}$ and $R_{\min }$ all depend on the underlying probability measure $P$. The intrinsic risk $R_{\min }$, although strictly less than the a priori risk $\operatorname{Var}[H]$, is still strictly positive and cannot be neglected. Only in the following scenario can we eliminate the risk completely, in analogy to what is promised by the Black-Scholes formula.

Suppose that only two cases appear with positive probability: Either the exchange rate goes up to some level $x^{+}$and the contingent claim $H$ assumes a corresponding value $h^{+}$, or the exchange rate goes down to some level $x^{-}$and $H$ assumes the value $h^{-}$. Thus,

$$
\begin{aligned}
& P\left[X_{1}=x^{+}, H=h^{+}\right]=p \\
& P\left[X_{1}=x^{-}, H=h^{-}\right]=1-p
\end{aligned}
$$

with $x^{-}<(1+r) \cdot x_{0}<x^{+}$and some $p \in(0,1)$. This allows us to determine two constants $\xi$ and $V_{0}$ such that

$$
P\left[H=(1+r) \cdot V_{0}+\xi \cdot\left(X_{1}-(1+r) \cdot X_{0}\right)\right]=1 .
$$

In fact, (3.12) reduces (3.13) to two linear equations for the two unknowns $\xi$ and $V_{0}$, and the solution is given by

$$
\begin{aligned}
\xi & =\frac{h^{+}-h^{-}}{x^{+}-x^{-}} \\
V_{0} & =\frac{1}{1+r} \cdot\left(h^{+} \cdot \frac{(1+r) \cdot x_{0}-x^{-}}{x^{+}-x^{-}}+h^{-} \cdot \frac{x^{+}-(1+r) \cdot x_{0}}{x^{+}-x^{-}}\right) .
\end{aligned}
$$

Thus, there is a linear dependence between the random variables $H$ and $X_{1}-(1+r) \cdot X_{0}$, and the linear regression becomes perfect: $R_{\min }=0$. In this 
case, there is no need to adjust the fair price $V_{0}$ by a risk premium because the risk has completely disappeared. In particular, we have

$$
C_{1}-C_{0}=0,
$$

i.e. the strategy becomes self-financing. The random variable $\eta_{1}$ is now known in advance and coincides with the constant $(1+r) \cdot \eta_{0}$. For the specific values in our introductory example, we recover the strategy which was described in Section 2.

Note that the optimal values $\xi$ and $V_{0}$ in (3.14) do not involve the probability parameter $p$; they are the same for any measure $\tilde{P}$ which preserves the binary structure (3.12) with some parameter $\tilde{p} \in(0,1)$. In particular, we are free to switch from $P$ to the measure $P^{*}$ with

$$
p^{*}=\frac{(1+r) \cdot x_{0}-x^{-}}{x^{+}-x^{-}}
$$

so that

$$
x_{0}=E^{*}\left[\frac{X_{1}}{1+r}\right] .
$$

Thus, the discounted exchange rate behaves like a fair game under $P^{*}$. In this new model, the fair price $V_{0}$ can now be computed directly, without going through the exercise of computing $\xi$ and $V_{0}$ from two linear equations. In fact, (3.15) implies

$$
E^{*}\left[V_{1}-\xi \cdot\left(X_{1}-(1+r) \cdot X_{0}\right)\right]=(1+r) \cdot V_{0},
$$

and by (3.3) and (3.16) we obtain

$$
V_{0}=E^{*}\left[\frac{H}{1+r}\right],
$$

as prescribed by Chr. Huygens and J. Bernoulli.

The preceding discussion of the binary scenario, viewed as a special case of the general two-period model, is just an elementary remark on the linear regression problem. But (3.17) is the exact analogue to the Black-Scholes formula. In both cases, the model is complete in the sense that any contingent claim $H$ can be generated by a suitable strategy as in (3.13). This allows us to reduce the risk to 0 , and to compute the value $V_{0}$ by an appropriate change of measure.

\section{HEDGING BY SEQUENTIAL REGRESSION}

Let us now consider a multiperiod model where the evolution of the exchange rate is given by a stochastic process $X_{k}(k=0, \ldots, T)$ on some probability space $(\Omega, \widetilde{F}, P)$. Let $\widetilde{F}_{k}$ denote the $\sigma$-field of events which are observable up to and including time $k$. We assume that $X_{k}$ is $\mathscr{F}_{k}$-measurable and square-integrable. In order to avoid complicated notations, we work again without interest rates. This 
is no restriction since we can always start by discounting the original price processes.

An option is described by a square-integrable random variable $H \in L^{2}(P)$; for example, a call option with strike $K$ would correspond to $H=\left(X_{T}-K\right)^{+}$. A trading strategy is given by two stochastic processes $\xi_{k}(k=1, \ldots, T)$ and $\eta_{k}$ $(k=0, \ldots, T) . \xi_{k}$ is the amount of US dollars held in period $k$ and has to be fixed at the beginning of that period, i.e. we assume that

$$
\xi_{k} \text { is } \mathscr{F}_{k-1} \text {-measurable }(k=1, \ldots, T) .
$$

The amount $\eta_{k}$ of Swiss francs in period $k$ can be chosen at the end of this period, i.e. we assume that

$$
\eta_{k} \text { is } \mathscr{F}_{k} \text {-measurable }(k=0, \ldots, T) \text {. }
$$

For such a trading strategy, the value of the portfolio at time $k$ is given by

$$
V_{k}=\xi_{k} \cdot X_{k}+\eta_{k}(k=1, \ldots, T),
$$

and $V_{0}=\eta_{0}$. We admit only strategies such that each $V_{k}$ is square-integrable and such that the contingent claim $H$ is produced in the end, i.e. we require

$$
V_{T}=H .
$$

Due to the flexibility allowed by (4.2), this can always be achieved by a suitable choice of $\eta r$. The cumulative cost at time $k$ is given by

$$
C_{k}=V_{k}-\sum_{j=1}^{k} \xi_{j} \cdot \Delta X_{j}
$$

with $\Delta X_{j}:=X_{j}-X_{j-1}$, and $C_{0}=V_{0}=\eta_{0}$. Going backwards from time $T$, we can now apply the argument of the preceding section step by step to determine our trading strategy recursively. Suppose that the random variables $\xi_{k+2}, \ldots, \xi_{T}$ and $\eta_{k+1}, \ldots, \eta_{T}$ (or, equivalently, $V_{k+1}, \ldots, V_{T}$ ) have already been prescribed. At time $k$, we want to choose first $\xi_{k+1}$ and then $V_{k}$ (respectively $\eta_{k}=V_{k}-\xi_{k} \cdot X_{k}$ ) such that the conditional risk

$$
\begin{aligned}
R_{k} & :=E\left[\left(C_{k+1}-C_{k}\right)^{2} \mid \tilde{\mathscr{F}}_{k}\right] \\
& =E\left[\left(V_{k+1}-V_{k}-\xi_{k+1} \cdot \Delta X_{k+1}\right)^{2} \mid \tilde{F}_{k}\right]
\end{aligned}
$$

is minimized. Note that the expectation in (3.6) has now been replaced by a conditional expectation. In analogy to (3.9), this implies

$$
C_{k}=E\left[C_{k+1} \mid \mathscr{F}_{k}\right] \text {, }
$$

i.e. the cost process $C_{k}(k=0, \ldots, T)$ is a martingale. Moreover, the conditional versions of (3.7) and (3.8) yield the recursion formulae

$$
\xi_{k}=\frac{\operatorname{Cov} \tilde{\pi}_{k-1}\left(H-\sum_{j=k+1}^{T} \xi_{j} \cdot \Delta X_{j}, \Delta X_{k}\right)}{\operatorname{Var}_{\tilde{F}_{k-1}}\left[\Delta X_{k}\right]}
$$


and

$$
\eta_{k}=E\left[H-\sum_{j=k+1}^{T} \xi_{j} \cdot \Delta X_{j} \mid \mathscr{F}_{k}\right]-\xi_{k} \cdot X_{k} .
$$

In particular, this recursion leads to the fair price $V_{0}=\eta_{0}$ of our option.

The structure of the optimal strategy becomes much more transparent if $P$ is a martingale measure, i.e. if $X_{k}(k=0, \ldots, T)$ is a martingale under $P$ :

$$
X_{k}=E\left[X_{k+1} \mid \tilde{\mathbb{F}}_{k}\right] \quad(k=0, \ldots, T-1) .
$$

By (4.7) and (4.5), it follows that the value process $V_{k}(k=0, \ldots, T)$ is also a martingale, hence of the form

$$
V_{k}=E\left[H \mid \mathscr{F}_{k}\right]
$$

due to (4.4). We can now give a more direct construction of the optimal strategy. To begin with, (4.11) determines $\eta_{k}=V_{k}-\xi_{k} \cdot X_{k}$ as soon as we know $\xi_{k}$. In order to compute $\xi_{k}$, we use the fact that $H$ can be written as

$$
H=V_{0}+\sum_{j=1}^{T} \xi_{j}^{H} \cdot \Delta X_{j}+L_{T}^{H}
$$

where $L_{k}^{H}(k=0, \ldots, T)$ is a martingale which is orthogonal to $X_{k}(k=0, \ldots, T)$ in the sense that

$$
E\left[\Delta L_{k}^{H} \cdot \Delta X_{k} \mid \mathscr{F}_{k-1}\right]=0 .
$$

This allows us to conclude that

$$
\xi_{k}=\xi_{k}^{H}(k=1, \ldots, T)
$$

is the optimal hedging strategy. In fact, (4.11) and (4.12) imply

$$
V_{k}=V_{0}+\sum_{j=1}^{k} \xi_{j}^{H} \cdot \Delta X_{j}+L_{k}^{H},
$$

hence

$$
R_{k-1}=E\left[\left(\Delta L_{k}^{H}\right)^{2} \mid \mathscr{F}_{k-1}\right]+\left(\xi_{k}-\xi_{k}^{H}\right)^{2} \cdot E\left[\left(\Delta X_{k}\right)^{2} \mid \mathscr{F}_{k-1}\right]
$$

due to (4.13), and this is minimized by the choice of (4.14).

Now consider the very special case where $H$ can actually be generated by $X_{k}$ $(k=0, \ldots, T)$ in the sense that

$$
H=V_{0}+\sum_{j=1}^{T} \xi_{j}^{H} \cdot \Delta X_{j},
$$

i.e. where $L_{T}^{H}=0$ in (4.12). Then (4.16) implies $R_{k}=0(k=0, \ldots, T)$, hence

$$
C_{k+1}=C_{k}=C_{0}
$$


due to (4.6). Thus, the optimal strategy is self-financing. By (4.3) and (4.15),

$$
\begin{aligned}
\eta_{k} & =V_{k}-\xi_{k} \cdot X_{k} \\
& =V_{k-1}+\xi_{k} \cdot\left(X_{k}-X_{k-1}\right)-\xi_{k} \cdot X_{k} \\
& =V_{k-1}-\xi_{k} \cdot X_{k-1} .
\end{aligned}
$$

In this special case, the process $\eta_{k}(k=0, \ldots, T)$ is predictable, i.e.

$$
\eta_{k} \text { is } \tilde{F}_{k-1} \text {-measurable }(k=1, \ldots, T) \text {. }
$$

This means that all the ingredients of the trading strategy can already be fixed at the beginning of each period. Note also that the strategy remains the same if we change the measure $P$ to any measure $\tilde{P}$ which is equivalent to $P$ because this preserves the structure (4.17). Thus, the preceding discussion is valid for any model $P$ which can be obtained by an equivalent change of measure from a martingale model $P^{*}$ satisfying (4.17). In such a case, the explicit use of $P^{*}$ permits a direct computation of the fair option price. In fact, (4.17) implies

$$
V_{0}=E^{*}[H] \text {, }
$$

in analogy to (3.17) and (2.5).

\section{OPTION TRADING AND STOCHASTIC CALCULUS}

Let us briefly comment on the extension of our previous discussion to a continuous-time setting where $X_{t}(0 \leqslant t \leqslant T)$ is a square-integrable semimartingale on some probability space $(\Omega, \mathscr{F}, P)$ with a right-continuous filtration $\mathscr{F}_{t}$ $(0 \leqslant t \leqslant T)$. We can use the same conceptual approach to the pricing and hedging of options as in the discrete-time case, but the technicalities are much more involved.

Here again, the situation is most transparent in the complete case where, in analogy to (4.17), our square-integrable contingent claim $H$ can be represented as

$$
H=V_{0}+\int_{0}^{T} \xi_{u}^{H} \mathrm{~d} X_{u}
$$

$P$-almost surely, i.e. as a stochastic integral of some predictable process $\xi_{t}^{H}$ $(0 \leqslant t \leqslant T)$ with respect to the basic semimartingale $X_{t}(0 \leqslant t \leqslant T)$. In this case, we take $\xi_{t}=\xi_{t}^{H}$ and determine $\eta_{t}=V_{t}-\xi_{t} \cdot X_{t}$ in such a way that

$$
V_{t}=V_{0}+\int_{0}^{t} \xi_{u} \mathrm{~d} X_{u} \quad(0 \leqslant t \leqslant T) .
$$

Then we have $V_{T}=H$, i.e. the strategy produces the contingent claim $H$ at the initial cost $C_{0}=V_{0}$, and the resulting cost process

$$
C_{t}=V_{t}-\int_{0}^{t} \xi_{u} \mathrm{~d} X_{u}
$$


satisfies

$$
C_{t}=C_{0}=V_{0} \quad(0 \leqslant t \leqslant T) .
$$

Thus, the strategy is self-financing, and $V_{0}$ is the fair price of $H$. Now assume that there exists an equivalent martingale measure $P^{*}$ which preserves the structure (5.1). Let us switch from $P$ to $P^{*}$; this could be done more explicitly by means of a Girsanov transformation. In this new model, the price can now be computed directly as the expected value

$$
V_{0}=E^{*}[H]
$$

of our contingent claim $H$. In the special situation of (2.3), any square-integrable contingent claim admits a representation (5.1); this is a variant of a fundamental representation theorem of $\mathrm{K}$. ITÔ (1951). Thus, the problem is completely solved, and for an option of the form $H=\left(X_{T}-K\right)^{+}$, the pricing formula (5.2) reduces to the Black-Scholes formula (2.5). For a detailed introduction to the general complete case, we refer to HARRISON and PLISKA $(1981,1983)$.

In the incomplete case, but under the assumption that $P$ is a martingale measure, the optimal trading strategy can be determined as in (4.14). Here the risk can be reduced to the intrinsic risk, but it can no longer be eliminated completely. This case has been worked out in FöLLMER and SONDERMANN (1986) and SCHWEIZER (1984).

In a general incomplete model where $X_{t}(0 \leqslant t \leqslant T)$ is a semimartingale but not a martingale, the situation becomes very delicate. Here it is no longer possible to compute the optimal strategy by a simple backwards recursion as in the discrete-time case above. But it is shown in SCHWEIZER (1988) that one can derive an optimality equation for the strategy which is based on the Doob-Meyer decomposition of the semimartingale $X$ into a martingale and a predictable process of finite variation. This non-linear stochastic optimality equation can be solved by means of a suitable Girsanov transformation. We refer to SCHWEIZER (1988) for further details.

\section{THE VALUATION OF A STOP-LOSS CONTRACT}

D. SONDERMANN (1988) has pointed out that the valuation of a stop-loss contract can be viewed as an exact analogue to the pricing of an option. Consider a stochastic cumulative claim process $S_{t}(0 \leqslant t \leqslant T)$, a deterministic cumulative premium process

$$
p(t)=\int_{0}^{t} \dot{p}(u) \mathrm{d} u \quad(0 \leqslant t \leqslant T)
$$

with $\dot{p}>0$, and the associated stochastic process

$$
X_{t}=S_{t}-p(t) \quad(0 \leqslant t \leqslant T) .
$$

We assume that $\left(S_{t}\right)$ is a Poisson process with fixed jump height and deterministic 
intensity $\lambda(t)$. This model is complete in the sense that any square-integrable $H$ can be represented in the form

$$
H=V_{0}+\int_{0}^{T} \xi_{u}^{H} \mathrm{~d} X_{u}
$$

In particular, this is true for the random variable $H=\left(S_{T}-c\right)^{+}$associated to a stop-loss contract. Now we consider an insurance strategy given by $\xi_{t}(0 \leqslant t \leqslant T)$ and $\eta_{t}(0 \leqslant t \leqslant T)$. Here, $\eta_{t}$ is the cash reserve held at time $t$, and $\xi_{t}$ represents a proportional reinsurance contract inducing the capital flow

$$
\int_{0}^{t} \xi_{u}\left(\mathrm{~d} S_{u}-\dot{p}(u) \mathrm{d} u\right)=\int_{0}^{t} \xi_{u} \mathrm{~d} X_{u}
$$

We take $\xi_{t}=\xi_{t}^{H}$, and we determine $\eta_{t}=V_{t}-\xi_{t} \cdot X_{t}$ by setting

$$
V_{t}=V_{0}+\int_{0}^{t} \xi_{u}^{H} \mathrm{~d} X_{u}
$$

As in the previous section, we conclude that this strategy is self-financing and produces the random payment $H$ at the terminal time $T$. The required initial cost $V_{0}$ can be computed directly as the expected value

$$
V_{0}=E^{*}[H]=E^{*}\left[\left(S_{T}-c\right)^{+}\right]
$$

if we switch from $P$ to the new model $P^{*}$ where the process $X_{t}(0 \leqslant t \leqslant T)$ is a martingale; this is achieved by choosing the rate $\lambda^{*}(t)=\dot{p}(t)$. Note that, in complete analogy to our discussion of currency options, the fair premium $V_{0}$ does not depend on the a priori rate $\lambda(t)$. For a more detailed account see SoNDERMANN (1988).

\section{REFERENCES}

BAChelier, L. "Théorie de la Spéculation", Ann. Sci, Ec. Norm. Sup. III-17 (1900), $21-86$. BERnOULI.I, J. "Ars coniectandi" (1713).

Black, F. and SCHOlEs, M. "The Pricing of Options and Corporate Liabilities", Journal of Political Economy 81 (1973), 637-659.

Cox, J. C. and Ross, S. A. "The Valuation of Options for Alternative Stochastic Processes", Journal of Financial Economics 3 (1976), 145-166.

Follmer, H. and SONDERMANN, D. "Hedging of Non-Redundant Contingent Claims", in: HiLdENBRAND, W. and MAS-Colell, A. (eds.), Contributions to Mathematical Economics NorthHolland (1986), 205-223.

Harrison, J. M. and Pliska, S. R. "Martingales and Stochastic Integrals in the Theory of Continuous Trading", Stochastic Processes and their Applications 11 (1981), 215-260.

Harrison, J. M. and Pliska, S. R. "A Stochastic Calculus Model of Continuous Trading: Complete Markets", Stochastic Processes and their Applications 15 (1983), 313-316.

Huygens, Chr. "De ratiociniis in ludo aleae" (1657).

ITÔ, K. "Multiple Wiener Integral", Journal of the Mathematical Society of Japan 3 (1951), $157-169$.

MERTON, R. C. "Theory of Rational Option Pricing", Bell Journal of Economics and Management Science 4 (1973), 141-183

Samuelson, P. A. "Rational Theory of Warrant Pricing", in: Cootner, P. H. (ed.), "The Random Character of Stock Market Prices”, MIT Press, Cambridge, Massachusetts (1964), 506-525.

ScHweiZER, M. "Varianten der Black-Scholes-Formel”, Diplomarbeit ETHZ, Zürich (1984). 
SCHWEIZER, M. "Hedging of Options in a General Semimartingale Model", Diss. ETHZ no. 8615, Zürich (1988).

SondermanN, D. "Reinsurance in Arbitrage-Free Markets", discussion paper no. B-82, University of Bonn (1988).

WienER, N. "Differential-Space", Journal of Mathematics and Physics 2 (1923), 131-174; reprinted in: WIENER, N. "Collected Works", Volume I, MIT Press (1976).

HANS Föllmer, MARTIN SCHWEIZER

Institut für Angewandte Mathernatik, Universität Bonn, Wegelerstr. 6, D-5300 Bonn 